\title{
Role of Dexmedetomidine in Early POCD in Patients Undergoing Thoracic Surgery
}

\author{
Jiao Ran $(\mathbb{D}$, Xiao Bai, Rurong Wang $(\mathbb{D}$, and Xuehan Li $(\mathbb{D}$ \\ Department of Anesthesiology, West China Hospital, Sichuan University \& the Research Units of West China (2018RU012), \\ Chinese Academy of Medical Sciences, China \\ Correspondence should be addressed to Rurong Wang; wangrurong@scu.edu.cn and Xuehan Li; xuehanli@scu.edu.cn
}

Received 31 August 2021; Accepted 1 November 2021; Published 23 November 2021

Academic Editor: Mian Peng

Copyright (c) 2021 Jiao Ran et al. This is an open access article distributed under the Creative Commons Attribution License, which permits unrestricted use, distribution, and reproduction in any medium, provided the original work is properly cited.

\begin{abstract}
Importance. Postoperative cognitive dysfunction (POCD) occurs in $6 \%-53 \%$ of elderly patients receiving major surgery and is related to longer hospital stays, increased hospital costs, and 1-year mortality. An increasing number of studies suggest that using dexmedetomidine (Dex) in critical care units is associated with reduced incidence of delirium. However, perioperative use of Dex for the prevention of POCD has not been well studied. Objective. To evaluate whether a low-dose perioperative infusion of Dex reduces early POCD. Design. This study was a double-blind, randomized, placebo-controlled trial that randomly assigned patients to Dex or saline placebo infused during surgery and patient-controlled intravenous analgesia (PCIA) infusion. Patients were assessed for postoperative cognitive decline. Interventions. Dex was infused at a loading dose of $0.5 \mu \mathrm{g} / \mathrm{kg}$ intravenously ( $15 \mathrm{~min}$ after entering the operation room) followed by a continuous infusion at a rate of $0.5 \mu \mathrm{g} / \mathrm{kg} / \mathrm{h}$ until one-lung ventilation or artificial pneumothorax ended. Patients in the Dex group received regular PCIA pump with additional dose of Dex $(200 \mu \mathrm{g})$. Results. In total, 126 patients were randomized, and 102 patients were involved in the result analysis. The incidence of POCD was 36.54\% (19/52) in the Dex group and 32.00\% (16/50) in the normal saline (NS) group, with no statistic difference. No significant difference was observed between the two groups in terms of Telephone Interview for Cognitive Status-Modified (TICS-m) scores at different times. However, the TICS-m score at 7 days after surgery was significantly lower than that at 30 days in 102 patients $(32.93 \pm 0.42$ vs. $33.92 \pm 0.47, P=0.03)$. The visual analogue scale scores in the Dex group were significantly lower than those in the NS group 1 day postoperation at rest and activity (2.00 [1.00-3.00] vs. 3.00 [2.00-4.00], $P<0.01 ; 4.00$ [3.00-5.00] vs. 5.00 [4.00-6.00], $P<0.05$, respectively). Patients receiving Dex or NS had no statistical difference in activities of daily living (ADLs) scores at 7 and 30 days after surgery, but the ADL score at 30 days after surgery showed a significant reduction compared with that at 7 days $(P<0.01)$. Patients in the Dex group had a shorter hospital length of stay $(15.26 \pm 3.77$ vs. $17.69 \pm 5.09, P=0.02)$ and less expenses $(52458.71 \pm 10649.30$ vs. $57269.03 \pm 9269.98, P=0.04)$ than those in the NS group. Conclusions. Low-dose Dex in the perioperative period did not reduce the incidence of early POCD in thoracic surgery. However, it relieved postoperative pain, decreased the hospitalization expenses, and shortened the length of stay.
\end{abstract}

\section{Introduction}

Since the emergence of neuropsychological tests to objectively assess cognitive changes after cardiac surgery in the 1980s, postoperative neurocognitive disorder associated with anesthesia and surgery has become the concern of anesthesiologists [1]. The incidence of postoperative cognitive dysfunction (POCD) ranges from $6 \%$ to $53 \%[2,3]$, varying with age, education level, comorbidity, and surgery. Moreover, inconsistent methodologies may result in the variation of its incidence $[4,5]$.
The underlying pathogenesis of POCD is the neuroinflammation and oxidative stress from anesthesia and surgery $[6,7]$. Surgical trauma provokes the release of proinflammatory cytokines. Neuroinflammation may impair neural cells and increase the peripheral blood levels of neuron damageassociated biochemical markers.

Thoracic surgery is accompanied with one-lung ventilation (OLV) with double-lumen endotracheal tube or two-lung ventilation with $\mathrm{CO}_{2}$ artificial pneumothorax. No matter which ventilation method is adopted, ventilator-induced lung injury 
is clinically significant, as it induces direct lung injury and systemic inflammation $[8,9]$. In addition, an imbalanced ventilation/perfusion ratio can directly decrease the cerebral oxygen saturation and harm the cognition-related region [10].

On the basis of the neuroinflammatory hypothesis involved in POCD, anti-inflammatory and antioxidative stress strategies are potential treatments for patients undergoing thoracic surgery [6]. Dexmedetomidine (Dex), a highly selective $\alpha 2$ adrenergic receptor agonist, is widely used for sedation and analgesia. Dex relieves stress from surgery and anesthesia via the noradrenergic system [11]. In animal and human studies, Dex can inhibit the release of proinflammatory cytokines [7]. $\mathrm{Wu}$ et al. found that Dex could attenuate the inflammatory response in thoracic surgery [12]. Pavone et al. thought that higher infusion rate and longer duration of drug administration may be more effective in improving postoperative cognitive function [13]. Su et al. indicated that prophylactic lowdose Dex infusion $(0.1 \mu \mathrm{g} / \mathrm{kg} / \mathrm{h}$, from intensive care unit admission on the day of surgery until $08: 00 \mathrm{~h}$ on postoperative day 1 ) could reduce the prevalence of postoperative delirium [14]. Nevertheless, other studies held the view that Dex did not prevent postoperative delirium [15]. Considering that Dex has a dose-dependent effect on POCD, we hypothesized that Dex administration during operation and patient-controlled intravenous analgesia (PCIA) with Dex could reduce the incidence of POCD in patients undergoing thoracic surgery.

\section{Methods}

The prospective, randomized, double-blind, and controlled study protocol was approved by the Ethics Committee of West China Hospital, Sichuan University [2019 (2019042508)] and registered in the Chinese Clinical Trials Registry (ChiCRTIOR-16008837). We obtained written informed consent from all participants.

2.1. Patients and Study Design. We recruited 126 patients aged 45 years or older who underwent elective thoracic surgery with American Society of Anesthesiologists class I-III from July 2019 to December 2019. Twelve nonoperative patients were included to evaluate the learning effects by repeated test of Repeatable Battery for the Assessment of Neuropsychological Status (RBANS). Patients would be excluded if they met any of the exclusion criteria: Telephone Interview for Cognitive StatusModified (TICS-m) score less than 28, a history of neurological disease, psychiatric and antidepression drugs, education level under primary school, inability to communicate because of dysaudia and vision disorder, serious hepatic dysfunction (Child-Pugh class C) and renal dysfunction (undergoing dialysis), sick sinus syndrome, severe sinus bradycardia ( $<50$ beats per min), or second- or third-degree atrioventricular block without pacemaker. The exit criteria were anesthesia duration less than $2 \mathrm{~h}$, inability to accomplish the cognitive assessment, and reoperation after surgery.

Patients were randomized into two groups: (1) Dex group, with a bolus of $0.5 \mu \mathrm{g} / \mathrm{kg}$ of Dex (Jiangsu Hengrui Medicine Co., Ltd., Jiangsu, China; specification $2 \mathrm{~mL}$ : $200 \mu \mathrm{g}$ ) (15 min after entering the operation room) followed by a continuous infusion at $0.5 \mu \mathrm{g} / \mathrm{kg} / \mathrm{h}$ until $\mathrm{OLV} / \mathrm{CO}_{2}$ artificial pneumothorax ended; (2) normal saline group (NS group), with NS administered as a bolus and a continuous infusion with identical volume and rate as the Dex group. A PCIA pump was used for postoperative analgesia in all patients. The PCIA protocol in the NS group was as follows: sufentanil $250 \mu \mathrm{g}+$ tramadol 500 $\mathrm{mg}+$ granisetron $9 \mathrm{mg}$. Patients in the Dex group received PCIA pump with an additional dose of Dex $(200 \mu \mathrm{g})$. The PCIA parameters were as follows: total amount of $200 \mathrm{~mL}$, background infusion rate of $2 \mathrm{~mL} / \mathrm{h}$, and bolus dose of $0.5 \mathrm{~mL}$, with a lock-out of $15 \mathrm{~min}$. The masked drugs were provided by the nurse who did not take part in other procedures.

2.2. Anesthesia Management. Patients received radial artery cannulation in the preanesthesia care unit, and arterial blood gas analysis was conducted before anesthesia $\left(t_{0}\right)$, before $\mathrm{OLV} / \mathrm{CO}_{2}$ artificial pneumothorax $\left(t_{1}\right), 20 \mathrm{~min}$ later $\left(t_{2}\right)$, and $20 \mathrm{~min}$ after the end of $\operatorname{OLV}\left(t_{3}\right)$. After admission to the operation room, pulse oxygen saturation $\left(\mathrm{SPO}_{2}\right)$, electrocardiogram, bi-spectral index (BIS), and regional cerebral oxygen saturation $\left(\mathrm{rSO}_{2}\right)$ were monitored. Anesthesia was induced with midazolam, sufentanil, propofol, and cisatracurium. Sevoflurane and remifentanil were used for maintenance with BIS $40-60$. A low tidal volume $(5-7 \mathrm{~mL} / \mathrm{kg})$ was applied during $\mathrm{OLV} / \mathrm{CO}_{2}$ artificial pneumothorax.

2.3. Neurocognitive Test. All patients were evaluated by the neuropsychological test battery (NTB; i.e., RBANS) [16] before surgery and 7 days after surgery/before discharge by a trained interviewer blinded to the treatment group. RBANS includes 12 subtests: list learning, story memory, figure copy, line orientation, picture naming, semantic fluency, digit span, coding, list recall, list recognition, story recall, and figure recall, which measure immediate memory, language, attention, visuospatial function, and delayed memory.

TICS-m was carried out before surgery and 7 days and 1 month postoperatively. TICS-m consists of 12 items that assess the cognitive function of immediate and delayed memory, orientation, language, calculation, and conceptual knowledge [17]. The total score is 50, and patients with higher scores have better cognitive function. The activities of daily living (ADLs) included instrument ADLs and basic ADLs with 14 items [18]. For each item, scoring from 1 to 4, higher scores, indicate poorer ADLs. The ADLs were assessed at the same time as the TICS-m postoperative test. Pain was evaluated daily in the first three days after surgery, and postoperative pain was graded by visual analogue scale (VAS).

2.4. Criteria for POCD Diagnosis. POCD was diagnosed according to the International Study of POCD1 definition [19]. The calculation formula is as follows:

$$
\begin{aligned}
Z & =\frac{\Delta x-\Delta x_{C}}{S D\left(\Delta x_{C}\right)}, \\
Z_{\text {combined }} & =\frac{\sum Z a, b, c, d, \text { etc }}{S D\left(\sum Z \text { control }\right)},
\end{aligned}
$$

where $\Delta x$ is the subtracted preoperative score from postoperative score in the operative group, $\Delta x_{C}$ is the subtracted 


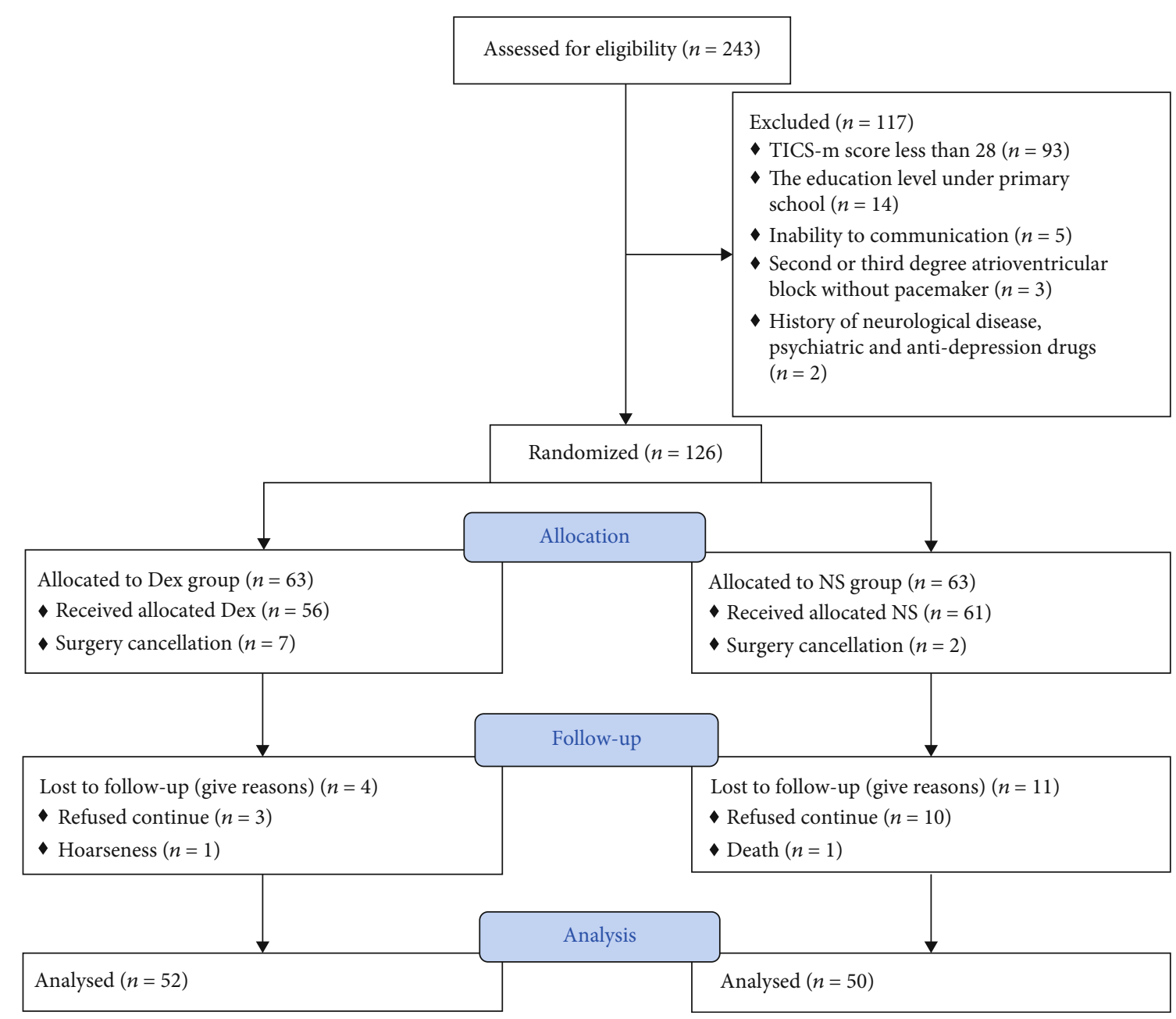

Figure 1: Enrolment flowchart.

preoperative score from postoperative score in the nonoperative group, $\Sigma Z$ is the sum of the $Z$ score in RBANS for each individual in the operative group, and $\Sigma Z$ control is the sum of the $Z$ score in RBANS in the nonoperative group.

POCD was defined as the $Z$ score less than -1.96 in at least two subtests or if the $Z_{\text {combined }}$ score was -1.96 or less.

2.5. Outcome. The primary outcome was the incidence of POCD, assessed with RBANS before surgery and 7 days after surgery/before discharge. The secondary outcomes included the scores of TICS-m before the surgery, 7 days and 30 days after the surgery. The ADL scores at 7 and 30 days after surgery and VAS scores during rest and activity from 1 to 3 days after surgery were also evaluated in this study. Other indicators including arterial blood gas at $t_{0}, t_{1}, t_{2}$, and $t_{3}$; cerebral regional oxygen saturation; duration of the surgery, anesthesia, and $\mathrm{OLV} / \mathrm{CO}_{2}$ artificial pneumothorax; hospital stay; and hospitalization expenses were analyzed as well.

2.6. Statistical Analysis. Sample size was based on a $46 \%$ incidence of POCD in thoracic surgery [20], and we assumed that Dex would reduce the incidence of POCD by $50 \%$ with $\alpha=0.05$ and $\beta=0.2$. We would require 126 patients to identify the difference with a two-tailed test.

Numerical variables were expressed as the mean \pm standard deviation or median (interquartile range). Categorical variables were shown as frequency (percentage). Continuous variables were analyzed using the $t$-test or Mann-Whitney $U$ test, as appropriate, and ranked data were analyzed using the Mann-Whitney $U$ test. The chi-square or Fisher's exact test was used for categorical variables. Repeated measurement data were analyzed by repeated measurement data analysis of variance. $P<0.05$ was considered statistically significant. Statistical analysis was performed using SPSS 24.0 (IBM Inc., Chicago, IL, USA).

\section{Results}

A total of 243 patients were screened. Among them, 126 patients were enrolled and randomly allocated to receive either Dex $(n=63)$ or NS $(n=63)$. A total of 52 patients in the Dex group and 50 patients in the NS group completed the RBANS at 7 days postoperatively or before discharge (Figure 1). The demographic and medical data are shown in Table 1 . No 
TABLE 1: Characteristics and intraoperative variables.

\begin{tabular}{|c|c|c|c|}
\hline & Dex group $(n=52)$ & NS group $(n=50)$ & $P$ \\
\hline Age (year) & $59.00 \pm 7.72$ & $62.08 \pm 7.51$ & 0.08 \\
\hline Sex (male, \%) & $29(55.77 \%)$ & $31(62.00 \%)$ & 0.52 \\
\hline BMI & $23.83 \pm 2.54$ & $23.83 \pm 2.54$ & 0.93 \\
\hline \multicolumn{4}{|l|}{ Educational level } \\
\hline Primary & $7(13.46 \%)$ & $5(10.00 \%)$ & \multirow{4}{*}{0.71} \\
\hline Junior high school & $12(23.08 \%)$ & $15(30.00 \%)$ & \\
\hline Senior high school & $14(26.92 \%)$ & $12(24.00 \%)$ & \\
\hline College degree & $19(36.54 \%)$ & $18(36.00 \%)$ & \\
\hline TICS-m score (baseline) & $34.27 \pm 3.79$ & $32.84 \pm 2.85$ & 0.14 \\
\hline Smoking history & $11(21.15 \%)$ & $16(32.00 \%)$ & 0.22 \\
\hline Drinking history & $5(9.62 \%)$ & $9(18.00 \%)$ & 0.22 \\
\hline Coronary heart disease & $0(0.00 \%)$ & $3(6.00 \%)$ & 0.11 \\
\hline Hypertension & $7(13.46 \%)$ & $15(30.00 \%)$ & 0.04 \\
\hline Diabetes & $2(3.85 \%)$ & $7(14.00 \%)$ & 0.09 \\
\hline Prolonged $\mathrm{rSO}_{2}$ desaturation ${ }^{*}$ & $0[0.00-0.79]$ & $0.02[0.00-5.12]$ & 0.27 \\
\hline \multicolumn{4}{|l|}{ Oxygenation index } \\
\hline T0 & $396.34 \pm 54.54$ & $409.9 \pm 64.71$ & 0.36 \\
\hline $\mathrm{T} 1$ & $396.00[323.75-494.25]$ & $401.00[361.00-442.00]$ & 0.86 \\
\hline $\mathrm{T} 2$ & $152.42 \pm 69.82$ & $174.30 \pm 81.91$ & 0.22 \\
\hline T3 & $321.26 \pm 103.84$ & $314.95 \pm 105.87$ & 0.80 \\
\hline Anesthesia duration (min) & $174.49 \pm 50.18$ & $162.51 \pm 42.58$ & 0.27 \\
\hline Surgery duration (min) & $106.76 \pm 52.07$ & $102.75 \pm 34.98$ & 0.69 \\
\hline OLV/artificial pneumothorax duration (min) & $80.0[65.0-99.5]$ & $87.0[69.0-112.2]$ & 0.44 \\
\hline Length of stay (day) & $15.26 \pm 3.77$ & $17.69 \pm 5.09$ & 0.02 \\
\hline The total hospital cost & $52458.71 \pm 10649.30$ & $57269.03 \pm 9269.98$ & 0.04 \\
\hline
\end{tabular}

BMI: body mass index; TICS-m: Telephone Interview for Cognitive Status-Modified; OLV: one lung ventilation; ${ }^{*} \mathrm{rSO}_{2}$ area under the curve (AUC) of desaturation below $20 \%$ of baseline or AUC of desaturation below $50 \%$ absolute value [21].

significant difference was observed in age, gender, and body mass index between the Dex and NS groups. Patients in the Dex group had a shorter hospital length of stay and less expenses than those in the NS group.

3.1. Primary Outcome. A total of 35 patients developed POCD at 7 days postoperatively or before discharge, including 19 in the Dex group (36.54\%) and 16 in the NS group (32.00\%) $(P=0.63)$ (Table 2).

The preoperative and postoperative/before discharge RBANS scores are listed in Table 3. No significant statistical difference was observed between the two groups $(P>0.05)$ (Table 3).

3.2. Secondary Outcomes. The different interventions, Dex or NS, had no influence on the TICS-m score at different times (intervention effect, $P \geq 0.05$ ) (Table 4). Compared with those in the NS group, the TICS-m scores were higher in the Dex group (intervention effect, $P=0.04$ ) (Table 4) (Figure 2). The TICS-m scores at different times were significantly different (time effect, $P=0.02$ ) (Table 4). Compared with that at 7
TABLE 2: The incidence of POCD.

\begin{tabular}{lccc}
\hline & Dex group $(n=52)$ & NS group $(n=50)$ & $P$ \\
\hline Incidence $(n, \%)$ & $19(36.54 \%)$ & $16(32.00 \%)$ & 0.63 \\
\hline
\end{tabular}

days postoperatively, the cognitive function improved at 1 month after surgery (Figure 3).

The VAS scores in the Dex group were significantly lower than those in the NS group 1 day postoperation at rest and activity (Figure 4). From the 2nd day after surgery, the VAS scores between the two groups were not statistically different.

Patients receiving Dex vs. NS had no statistical difference in ADL scores at 7 and 30 days after surgery, but the ADL scores at 30 days after surgery were significantly reduced compared with those at 7 days (Figure 5).

\section{Discussion}

In our study, we found that Dex did not reduce the incidence of early POCD in thoracic surgery. POCD developed in $36.54 \%(19 / 52)$ of patients in the Dex group and $32.00 \%$ 
TABLE 3: RBANS scores in both groups.

\begin{tabular}{|c|c|c|c|c|c|c|}
\hline & \multicolumn{3}{|c|}{ Preoperation } & \multicolumn{3}{|c|}{ Postoperation } \\
\hline & Dex group & NS group & $P$ & Dex group & NS group & $P$ \\
\hline List learning & $22.92 \pm 4.82$ & $22.38 \pm 3.87$ & 0.53 & $27.65 \pm 5.08$ & $27.22 \pm 4.84$ & 0.66 \\
\hline Story memory & $12.92 \pm 4.47$ & $13.08 \pm 3.85$ & 0.85 & $15.54 \pm 4.75$ & $16.02 \pm 4.24$ & 0.59 \\
\hline Figure copy & $15.29 \pm 2.84$ & $14.64 \pm 3.00$ & 0.27 & $14.02 \pm 2.85$ & $13.78 \pm 2.76$ & 0.67 \\
\hline Line orientation & $15.65 \pm 2.92$ & $14.98 \pm 2.87$ & 0.24 & $15.62 \pm 3.11$ & $15.52 \pm 2.76$ & 0.87 \\
\hline Picture naming & $9.33 \pm 1.04$ & $9.14 \pm 1.13$ & 0.39 & $9.42 \pm 0.87$ & $9.42 \pm 0.86$ & 0.99 \\
\hline Semantic fluency & $19.42 \pm 3.67$ & $19.14 \pm 4.50$ & 0.73 & $19.35 \pm 3.38$ & $18.10 \pm 3.82$ & 0.08 \\
\hline Digit span & $12.60 \pm 2.58$ & $11.98 \pm 2.79$ & 0.25 & $12.52 \pm 2.59$ & $11.50 \pm 2.79$ & 0.06 \\
\hline Coding & $36.83 \pm 9.34$ & $33.50 \pm 9.70$ & 0.08 & $35.94 \pm 10.44$ & $33.74 \pm 10.22$ & 0.29 \\
\hline List recall & $3.85 \pm 2.35$ & $4.00 \pm 2.46$ & 0.75 & $6.04 \pm 2.44$ & $5.34 \pm 2.75$ & 0.18 \\
\hline List recognition & $18.83 \pm 2.12$ & $18.86 \pm 1.78$ & 0.93 & $19.23 \pm 1.69$ & $19.24 \pm 1.36$ & 0.98 \\
\hline Story recall & $6.71 \pm 2.23$ & $6.48 \pm 2.60$ & 0.63 & $8.02 \pm 2.46$ & $7.34 \pm 3.01$ & 0.22 \\
\hline Figure recall & $11.79 \pm 3.71$ & $11.02 \pm 4.19$ & 0.33 & $12.44 \pm 3.58$ & $11.76 \pm 4.19$ & 0.38 \\
\hline
\end{tabular}

TABLE 4: The TICS-m scores between both groups.

\begin{tabular}{|c|c|c|c|c|c|c|}
\hline \multirow{2}{*}{ Group } & \multicolumn{3}{|c|}{ Time } & \multicolumn{3}{|c|}{$P$} \\
\hline & Preoperative & Postoperative 7 days & Postoperative 1 month & Interactive effect & Time effect & Intervention effect \\
\hline Dex group & $34.05 \pm 4.06$ & $33.69 \pm 4.11$ & $34.97 \pm 3.54$ & \multirow{2}{*}{0.74} & \multirow{2}{*}{0.02} & \multirow{2}{*}{0.04} \\
\hline NS group & $32.85 \pm 2.81$ & $32.26 \pm 3.29$ & $33.18 \pm 4.29$ & & & \\
\hline
\end{tabular}

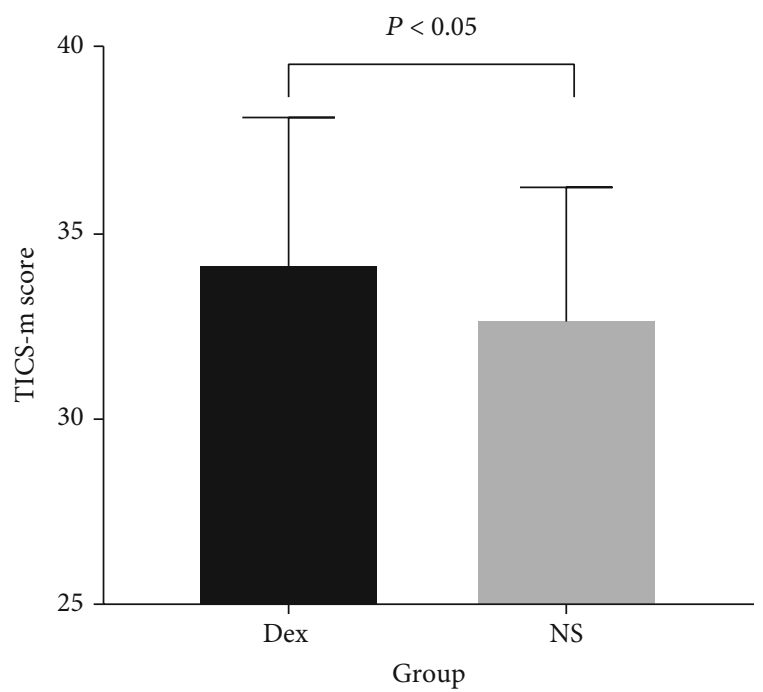

FIGURE 2: Tics-m score in both groups.

$(16 / 50)$ of patients in the NS group. No significant statistical and clinical difference was observed between the two groups. The incidence of POCD 7 days after surgery ranges from $17 \%$ to $40 \%$ in patients with noncardiac surgery $[19,22$, 23]. In thoracic surgery, special ventilation methods induce direct lung injury and systemic inflammation and aggravate postoperative cognitive decline. Egawa et al. found that the incidence of POCD was higher in thoracic surgery, and the incidence was $33.3 \%$ and $22.2 \%$ in patients undergoing tho-

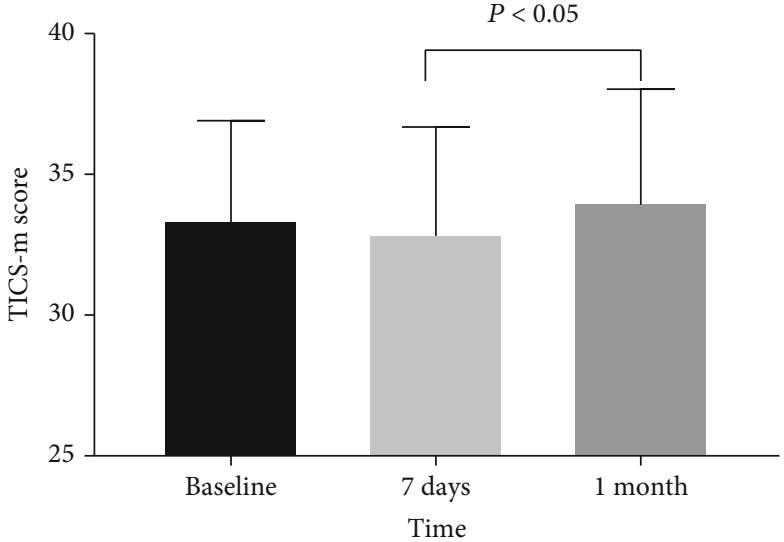

FIgURE 3: Tics-m score at different times.

racic surgery with sevoflurane and propofol anesthesia, respectively [24].

Our results showed that low-dose Dex in the perioperative period could not improve early cognitive function after thoracic surgery. This finding was in line with a multicenter prospective trial, which was unable to demonstrate a benefit of Dex in POD and POCD and prematurely terminated for futility [15]. In this study, Dex was infused at $0.5 \mu \mathrm{g} / \mathrm{kg} / \mathrm{h}$ during surgery and up to $2 \mathrm{~h}$ in the recovery room. Some studies $[13,14,25,26]$ hold the view that low-dose Dex could improve cognitive function over a long period. In our study, patients received a loading dose of Dex $(0.5 \mu \mathrm{g} /$ 

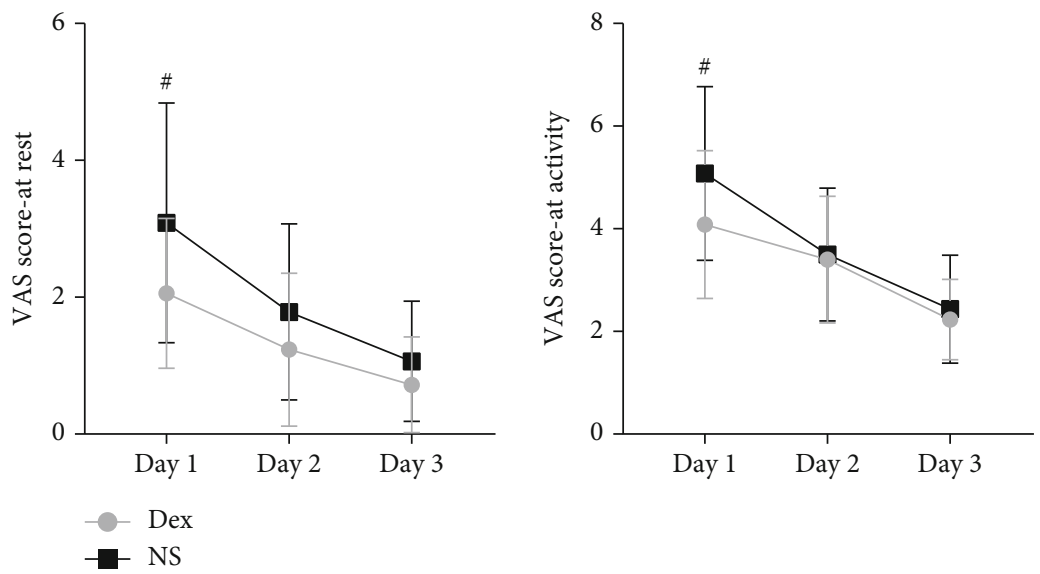

Figure 4: The VAS score after surgery at rest and activity in both groups. ${ }^{\#} P<0.05$, compared with the NS group.

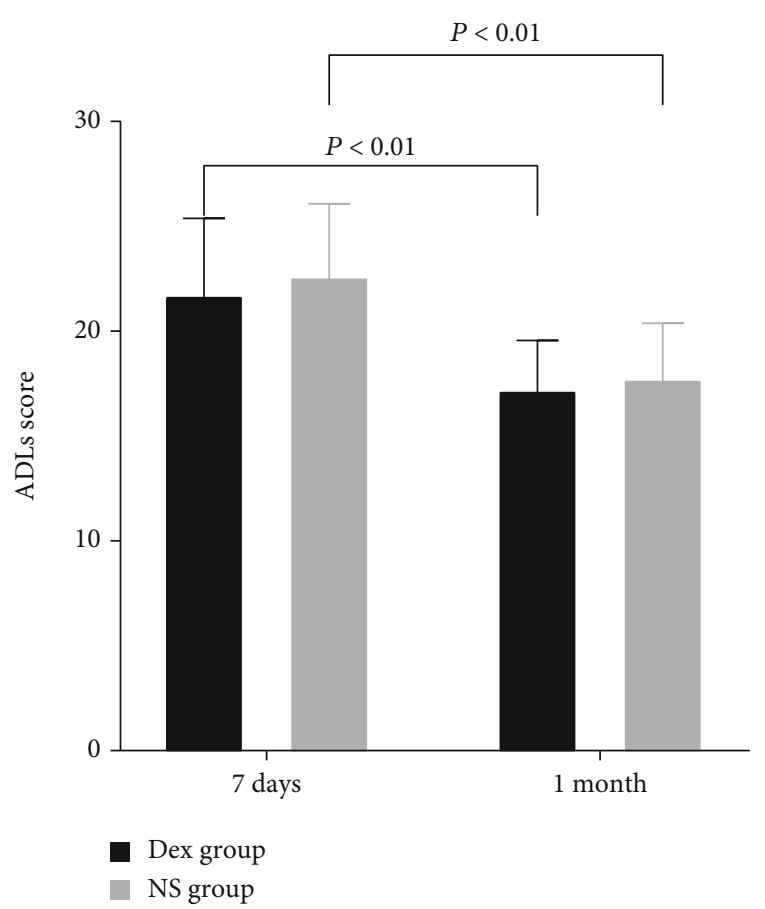

FIGURE 5: The ADL score in both groups 7 days and 1 month after surgery.

$\mathrm{kg}$ intravenously, $15 \mathrm{~min}$ after entering the operation room) followed by a continuous infusion at a rate of $0.5 \mu \mathrm{g} / \mathrm{kg} / \mathrm{h}$ until OLV or artificial pneumothorax ended. Moreover, the patients in the Dex group received a regular PCIA pump with an additional dose of Dex $(200 \mu \mathrm{g})$. However, our results showed that Dex could not reduce the incidence of POCD. This may be related to the lower concentration of Dex used in postoperative PCIA. Some studies thought that Dex only suppressed the symptom of agitation in patients undergoing thoracic surgery, rather than improving their cognition [27].

Meanwhile, the incidence of POCD varies with the diagnosis method. The Nomenclature Consensus Working Group recommends that objective cognition decline requires an NTB. So far, there is no unified NTB. As a kind of NTB,
RBANS can be used for the repeated measurement of cognitive function, and its validity and test-retest reliability have been determined [28]. Some studies thought that Dex could improve the cognitive decline after surgery by Mini-Mental State Examination (MMSE) and Montreal Cognitive Assessment (MoCA) $[29,30]$. In a meta-analysis, Man et al. found that Dex could improve cognitive function, but the subgroup analysis, which included studies using NTB other than MMSE, found that Dex could not improve postoperative cognitive function [31]. Compared with MMSE and MoCA, RBANS is more comprehensive and sensitive to cognitive function. MMSE generally used to screen and has a ceiling effect. MoCA ignores the cultural difference when translating.

In the telephone follow-up, we found that 102 patients had better cognitive function at 1 month after surgery than 7 days after surgery. However, compared with the NS group, Dex could not improve the cognitive function and ADLs at 7 days and 1 month after surgery.

Finally, we found that Dex could relieve the postoperative pain at the first day after thoracic surgery, shorten the hospital stay, and reduce the hospitalization cost. The possible reasons are as follows: (1) postoperative analgesia can promote patients' early activities; enable patients to effectively cough and discharge secretions; and reduce complications such as atelectasis, pneumonia, and deep vein thrombosis. It plays an important role in rapid recovery after thoracic surgery [32]. (2) Dex may reduce opioid requirements, mitigating opioidrelated side effects such as nausea and vomiting and respiratory depression [33]. (3) Kim et al. found that Dex reduced emergence agitation in patients undergoing thoracic surgery [27]. It was beneficial to chest drainage. Whether intravenous use of Dex during the perioperative period can promote the early recovery of patients undergoing thoracic surgery requires further clinical research.

This study has several limitations. First, the sample size was small, because many patients could not complete the repeated cognition evaluation, especially the elderly and the less-educated patients. Second, patients were not categorized based on age. The applicability of this conclusion needs further research in elderly thoracic surgery patients. Finally, the long-term cognitive function was not evaluated. 
Complex mechanisms and factors are involved in cognitive function. It is difficult to improve patient's cognitive function only by medical treatments. Some studies have shown that multidisciplinary interventions, such as physical exercise; cognitive function training; and improvement of sleep, postoperative anxiety, and depression, may be beneficial for POCD $[23,34,35]$.

\section{Conclusion}

Low-dose Dex in the perioperative period did not reduce the incidence of early POCD in thoracic surgery. However, it relieved postoperative pain, decreased hospitalization expenses, and shortened the length of stay.

\section{Data Availability}

Data for the study can be found in the study. More information concerning the data can be obtained from the corresponding authors.

\section{Conflicts of Interest}

The authors declare that they have no conflicts of interest.

\section{Authors' Contributions}

Jiao Ran and Xiao Bai collected and analyzed the data, interpreted the data, and wrote and edited the manuscript. Xuehan $\mathrm{Li}$ and Rurong Wang designed the study and revised the manuscript. Jiao Ran and Xiao Bai equally contributed to the manuscript.

\section{References}

[1] J. A. Savageau, B. A. Stanton, C. D. Jenkins, and M. D. Klein, "Neuropsychological dysfunction following elective cardiac operation. I. Early assessment," Survey of Anesthesiology, vol. 27, no. 4, p. 238, 1983.

[2] T. G. Monk, B. C. Weldon, C. W. Garvan et al., "Predictors of cognitive dysfunction after major noncardiac surgery," Anesthesiology, vol. 108, no. 1, pp. 18-30, 2008.

[3] M. F. Newman, J. L. Kirchner, and B. Phillips-Bute, "Longitudinal assessment of neurocognitive function after coronaryartery bypass surgery," The New England Journal of Medicine, vol. 344, pp. 81-82, 2001.

[4] L. Evered, B. Silbert, D. S. Knopman et al., "Recommendations for the nomenclature of cognitive change associated with anaesthesia and surgery-2018," British Journal of Anaesthesia, vol. 121, no. 5, pp. 1005-1012, 2018.

[5] E. Symes, P. Maruff, A. Ajani, and J. Currie, "Issues associated with the identification of cognitive change following coronary artery bypass grafting," Australian \& New Zealand Journal of Psychiatry, vol. 34, no. 5, pp. 770-784, 2015.

[6] S. A. Safavynia and P. A. Goldstein, "The role of neuroinflammation in postoperative cognitive dysfunction: moving from hypothesis to treatment," Frontiers in Psychiatry, vol. 9, p. 752, 2018.

[7] D. R. Skvarc, M. Berk, L. K. Byrne et al., "Post-operative cognitive dysfunction: an exploration of the inflammatory hypothe- sis and novel therapies," Neuroscience and Biobehavioral Reviews, vol. 84, pp. 116-133, 2018.

[8] R. Tomasi and V. von Dossow-Hanfstingl, "Critical care strategies to improve neurocognitive outcome in thoracic surgery," Current Opinion in Anaesthesiology, vol. 27, no. 1, pp. 44-48, 2014.

[9] M. Lin, Y. Shen, H. Wang et al., "A comparison between two lung ventilation with $\mathrm{CO}_{2}$ artificial pneumothorax and one lung ventilation during thoracic phase of minimally invasive esophagectomy," Journal of Thoracic Disease, vol. 10, no. 3, pp. 1912-1918, 2018.

[10] L. Tang, R. Kazan, R. Taddei, C. Zaouter, S. Cyr, and T. M. Hemmerling, "Reduced cerebral oxygen saturation during thoracic surgery predicts early postoperative cognitive dysfunction," British Journal of Anaesthesia, vol. 108, no. 4, pp. 623-629, 2012.

[11] Z. J. Carr, T. J. Cios, K. F. Potter, and J. T. Swick, "Does dexmedetomidine ameliorate postoperative cognitive dysfunction? A brief review of the recent literature," Current Neurology and Neuroscience Reports, vol. 18, no. 10, p. 64, 2018.

[12] C.-Y. Wu, Y.-F. Lu, M.-L. Wang et al., "Effects of Dexmedetomidine Infusion on Inflammatory Responses and Injury of Lung Tidal Volume Changes during One-Lung Ventilation in Thoracoscopic Surgery: A Randomized Controlled Trial," Mediators of Inflammation, vol. 2018, 8 pages, 2018.

[13] K. J. Pavone, P. Z. Cacchione, R. C. Polomano, L. Winner, and P. Compton, "Evaluating the use of dexmedetomidine for the reduction of delirium: an integrative review," Heart \& Lung, vol. 47, no. 6, pp. 591-601, 2018.

[14] X. Su, Z.-T. Meng, X.-H. Wu et al., "Dexmedetomidine for prevention of delirium in elderly patients after non- cardiac surgery: a randomised, double-blind, placebo-controlled trial," The Lancet, vol. 388, no. 10054, pp. 1893-1902, 2016.

[15] S. Deiner, X. Luo, H. M. Lin et al., "Intraoperative infusion of dexmedetomidine for prevention of postoperative delirium and cognitive dysfunction in elderly patients undergoing major elective noncardiac Surgery," JAMA Surgery, vol. 152, no. 8, article e171505, 2017.

[16] C. Randolph, M. C. Tierney, E. Mohr, and T. N. Chase, "The Repeatable Battery for the Assessment of Neuropsychological Status (RBANS): preliminary clinical validity," Journal of Clinical and Experimental Neuropsychology, vol. 20, no. 3, pp. 310319, 1998.

[17] E. H. Seo, D. Y. Lee, S. G. Kim et al., "Validity of the telephone interview for cognitive status (TICS) and modified TICS (TICSm) for mild cognitive imparment (MCI) and dementia screening," Archives of Gerontology and Geriatrics, vol. 52, no. 1, pp. e26-e30, 2011.

[18] M. E. Mlinac and M. C. Feng, "Assessment of activities of daily living, self-care, and independence," Archives of Clinical Neuropsychology, vol. 31, no. 6, pp. 506-516, 2016.

[19] J. T. Moller, P. Cluitmans, L. S. Rasmussen et al., "Long-term postoperative cognitive dysfunction in the elderly: ISPOCD1 study," The Lancet, vol. 351, no. 9106, pp. 857-861, 1998.

[20] K. Y. Wang, Q. Y. Yang, P. Tang, H. X. Li, H. W. Zhao, and $\mathrm{X}$. B. Ren, "Effects of ulinastatin on early postoperative cognitive function after one-lung ventilation surgery in elderly patients receiving neoadjuvant chemotherapy," Metabolic Brain Disease, vol. 32, no. 2, pp. 427-435, 2017.

[21] Z. Colak, M. Borojevic, A. Bogovic, V. Ivancan, B. Biocina, and V. Majeric-Kogler, "Influence of intraoperative cerebral 
oximetry monitoring on neurocognitive function after coronary artery bypass surgery: a randomized, prospective study [J]," European Journal of Cardio-Thoracic Surgery, vol. 47, no. 3, pp. 447-454, 2015.

[22] B. Silbert, L. Evered, D. A. Scott, and S. McMahon, "Preexisting cognitive impairment is associated with postoperative cognitive dysfunction after hip joint replacement surgery," Anesthesiology, vol. 122, no. 6, pp. 1224-1234, 2015.

[23] S. Deiner, X. Liu, H. M. Lin et al., "Subjective cognitive complaints in patients undergoing major non-cardiac surgery: a prospective single centre cohort trial," British Journal of Anaesthesia, vol. 122, no. 6, pp. 742-750, 2019.

[24] J. Egawa, S. Inoue, and T. Nishiwada, "Effects of anesthetics on early postoperative cognitive outcome and intraoperative cerebral oxygen balance in patients undergoing lung surgery: a randomized clinical trial," Canadian Journal of Anesthesial Journal canadien d'anesthésie, vol. 63, p. 116211169, 2016.

[25] X. Q. Cheng, B. Mei, Y. M. Zuo et al., "Retracted:A multicentre randomised controlled trial of the effect of intra-operative dexmedetomidine on cognitive decline after surgery," Anaesthesia, vol. 74, no. 6, pp. 741-750, 2019.

[26] C. Lee, C. H. Lee, G. Lee, M. Lee, and J. Hwang, "The effect of the timing and dose of dexmedetomidine on postoperative delirium in elderly patients after laparoscopic major noncardiac surgery: a double blind randomized controlled study," Journal of Clinical Anesthesia, vol. 47, pp. 27-32, 2018.

[27] J. A. Kim, H. J. Ahn, M. Yang, S. H. Lee, H. Jeong, and B. G. Seong, "Intraoperative use of dexmedetomidine for the prevention of emergence agitation and postoperative delirium in thoracic surgery: a randomized-controlled trial," Canadian journal of anaesthesia, vol. 66, no. 4, pp. 371-379, 2019.

[28] L. A. Evered and B. S. Silbert, "Postoperative cognitive dysfunction and noncardiac surgery," Anesthesia and Analgesia, vol. 127, no. 2, pp. 496-505, 2018.

[29] W. Yang, L. S. Kong, X. X. Zhu, R. X. Wang, Y. Liu, and L. R. Chen, "Effect of dexmedetomidine on postoperative cognitive dysfunction and inflammation in patients after general anaesthesia: a PRISMA-compliant systematic review and meta-analysis," Medicine, vol. 98, no. 18, article e15383, 2019.

[30] Y. Ge, Q. Li, Y. Nie et al., "Dexmedetomidine improves cognition after carotid endarterectomy by inhibiting cerebral inflammation and enhancing brain-derived neurotrophic factor expression," The Journal of international medical research., vol. 47, no. 6, pp. 2471-2482, 2019.

[31] Y. Man, Z. Guo, J. Cao, and W. Mi, "Efficacy of perioperative dexmedetomidine in postoperative neurocognitive function: a meta-analysis," Clinical and experimental pharmacology \& physiology, vol. 42, no. 8, pp. 837-842, 2015.

[32] T. J. P. Batchelor, N. J. Rasburn, E. Abdelnour-Berchtold et al., "Guidelines for enhanced recovery after lung surgery: recommendations of the Enhanced Recovery After Surgery (ERAS ${ }^{\circledR}$ ) Society and the European Society of Thoracic Surgeons (ESTS)," European Journal of Cardio-Thoracic Surgery, vol. 55, no. 1, pp. 91-115, 2019.

[33] C. Thompson, D. G. French, and I. Costache, "Pain management within an enhanced recovery program after thoracic surgery," Journal of Thoracic Disease, vol. 10, Supplement 32, pp. S3773-S3s80, 2018.
[34] J. Jiang, A. J. Fiocco, X. Cao et al., "The moderating role of COMT and BDNF polymorphisms on transfer effects following multi- and single-domain cognitive training among community-dwelling Shanghainese older adults," Frontiers in Aging Neuroscience, vol. 10, p. 198, 2018.

[35] O. C. Küster, D. Laptinskaya, P. Fissler et al., "Novel bloodbased biomarkers of cognition, stress, and physical or cognitive training in older adults at risk of dementia: preliminary evidence for a role of BDNF, irisin, and the kynurenine pathway," Journal of Alzheimer's disease : JAD., vol. 59, no. 3, pp. 1097-1111, 2017. 